\title{
Developments in Quasi-Optical Design for THz
}

\author{
Créidhe O’Sullivan ${ }^{* a}$, J. Anthony Murphy ${ }^{\text {a }}$, Gary Cahill ${ }^{\text {a }}$, Marcin Łukasz Gradziel ${ }^{\text {a }}$, Neil Trappe ${ }^{\text {a }}$, \\ David White $^{\mathrm{a}, \mathrm{b}}$, Vladimir Yurchenko ${ }^{\text {a }}$, Stafford Withington ${ }^{\mathrm{c}}$, Willem Jellema ${ }^{\mathrm{d}}$ \\ ${ }^{a}$ Department of Experimental Physics, NUI Maynooth, Co. Kildare, Ireland; \\ ${ }^{\mathrm{b}}$ Computing Department, Tallaght IT, Dublin 24, Ireland; \\ ${ }^{c}$ Cavendish Laboratory, Madingley Road, Cambridge, UK; \\ ${ }^{\mathrm{d}}$ SRON, P.O. Box 800, 9700 AV Groningen, The Netherlands
}

\begin{abstract}
Optical design in the terahertz $(\mathrm{THz})$ waveband suffers from a lack of dedicated software tools for modelling the range of electromagnetic and quasi-optical propagation conditions encountered in typical systems. Optical engineers are forced to use packages written for very different wavelength systems and there is often a lack of confidence in the results because of possible inappropriate underlying physical models. In this paper we describe the analytical techniques and dedicated CAD software tools (MODAL ${ }^{1}$ ) that we are developing for long-wavelength design and analysis in the $\mathrm{THz}$ waveband. Our basic approach to modelling long-wavelength propagation is the application of modal analysis appropriate to the problem under investigation ${ }^{2,3}$. We have extended this to include the efficient description of common off-axis (tilted) components such as simple curved reflectors ${ }^{4}$. In earlier research we have investigated the conditions under which approximate methods (ray tracing, paraxial modes) can provide extremely efficient and accurate solutions and situations where a more rigorous approach is required ${ }^{5,6}$. As a rigorous model of electromagnetic wave propagation, physical optics can be used to characterize complete systems to high accuracy. However, the straightforward approach is computationally intensive and, therefore, not suitable for the initial design or preliminary analysis of large multi-element optical systems. In order to improve the computational efficiency of the usual PO approach we have developed fast physical optics software, initially for the analysis of the ESA PLANCK system $^{7}$. The MODAL code is modular and multi-platform, and different propagation models can be used within the same framework. Distributed parallel computing enables significant reduction of the time needed to perform the calculations. We present the new software and analyses of the QuaD $^{8}$ and Herschel (HIFI) ${ }^{9}$ telescope systems.
\end{abstract}

Keywords: quasi-optics, physical optics, Gaussian beam modes, optical design

\section{INTRODUCTION}

There is currently considerable interest in the modelling and design of astronomical THz/submillimetre systems such as ALMA and ESA's PLANCK and Herschel Space Observatory. At these frequencies radiation in an astronomical receiver is often propagated and analysed as free-space beams. Unlike traditional optics however, these beams are only a few wavelengths in diameter and diffraction effects can become important. So THz optical systems are unique, requiring a different approach to those commonly used at visible wavelengths. Despite this, there is a lack of dedicated software tools for modelling the range conditions typically encountered and many such systems are still designed using optical ray-tracing techniques.

In previous papers ${ }^{5,6}$ the ability of a range of commercial optical design software packages (GLAD, ASAP, CODE V, Zemax) to analyse the behaviour of submillimetre optical systems was investigated. Although these packages are not specifically intended for use at submillimetre wavelengths, they represent the only classes of commercial optical design tools available with some diffraction capability. Originally we took the physical optics (PO) package, GRASP, an extremely powerful software tool for reflector antenna design and analysis, as our benchmark software against which the results of the other optical packages could be compared. An experimental verification of some of the GRASP results

\footnotetext{
Tel: +353-1-7083953, Fax: +353-1-7083313, creidhe.osullivan@may.ie
} 
has also been published ${ }^{10}$. It should be noted that GRASP, because of its complexity and computational intensity, is more suited to the verification phase rather than the instrument design phase and the rigorous PO technique becomes prohibitively slow for telescopes with large mirrors operating at high frequencies. As an alternative, a dedicated fast PO simulation method $^{7}$ has been specifically designed for large multi-beam multi-reflector systems with broadband channels and multi-moded feed horns. The method uses efficient integration over reflecting surfaces and the analytical propagation of TE/TM electromagnetic modes from the horns. It allows us to perform PO simulations of the main beams of large telescopes, such as PLANCK, for both mono-mode mono-frequency channels and broad-band polarization-averaged multi-mode channels at higher frequencies.

In this paper we will first briefly discuss some of the fundamental approximations that are inherent in the theoretical methods on which many current software packages are based. We then describe our new software package 'MODAL' that we are developing as a tool for quasi-optical instrument designers.

\section{LIMITATIONS OF CURRENT TECHNIQUES}

Optical design is essentially concerned with the problem of calculating an electromagnetic field over a surface in an optical system when the field, or currents, over some other surface is known. Techniques such as the Method of Moments attempt to calculate the current distribution over a surface precisely ${ }^{11}$ but the full solution to Maxwell's equations is usually extremely difficult to find and in practice approximations have to be made.

In general terms the field over an input surface is a vector field, and it is necessary to calculate the full vector field that results. In some cases, considering only one component of the field leads to relatively simple scalar solutions. For THz systems the question of whether a vector or a scalar solution is sought is intrinsically related to whether the field is of a paraxial or wide-angle nature.

Propagating the field onto the next optical component (solving the wave equation) requires diffraction integrals to be calculated for each field point calculated. Kirchhoff's approximation is the arithmetic average of the rigorous solutions when there is ambiguity over which vector quantity $(\mathbf{E}$ or $\mathbf{H})$ is being analysed. The averaging process appears in the form of the well-known $(1+\cos \theta) / 2$ obliquity term. Rather than evaluating diffraction integrals directly, it is possible to decompose an assumed source field into modes and then propagate the modes as required. Propagating modes through free space is usually straightforward and often simply consists of slipping the mode phases with respect to each other. Many commercial software packages use modal techniques. GLAD, for example, breaks the field down into plane waves $^{12}$. Gaussian modes, which are solutions of the paraxial wave equation ${ }^{13}$, allow a field distribution to be traced using only the scale size of the beam, the large-scale radius of curvature of the phase front and the phase slippage between modes. We often use Hermite-Gaussian modes for the analysis of paraxial systems with no particular axial symmetry. In the Gabor approach ${ }^{14}$, a field is decomposed into a discrete set of Gaussian beams shifted both laterally and in phase slope. A subset of these modes is used by the software package Zemax for its diffraction calculations. A plane wave analysis has the significant advantage that it is not limited to paraxial fields.

At optical wavelengths, away from boundary shadows and abrupt changes in intensity distribution, energy can be considered to be transported along curves, or light rays, obeying certain geometrical laws. For system design at visible wavelengths this technique is widely used and ray-tracing packages, such as CODE V and Zemax, have proved to be very successful. Ray-tracing can also be accurate for systems that are highly over-moded. In the submillimetre, however, the wavelength is typically an appreciable fraction of component sizes and so cannot be neglected. Systems tend to be at most few-moded and often have a compact optical layout. In this regime diffraction effects are important and the approach of geometrical optics is inadequate.

As an example consider QUaD, a ground-based high-resolution instrument designed to map CMB polarisation ${ }^{8,15}$. QUaD is a Cassegrain telescope whose basic design (Fig. 1) was developed using ray-tracing. Because of the Cassegrain design, the centres of beams propagating from the feed horn are reflected back from the secondary and could re-enter the lens system through the cryostat window. In order to reduce the amount of power reflected back into the system, a hole is cut in the centre of the secondary. According to a ray-tracing analysis this hole in the secondary can be 
designed so that its geometric shadow exactly matches the hole in the primary mirror and an on-axis beam reflected back from the secondary should ideally miss the cryostat. In fact, using a Gaussian beam or plane-wave mode approach to model diffraction shows that some power is produced in the geometric shadow of the hole. This 'Poisson spot' effect is not predicted by ray-tracing. Fig. 2 shows the result of an analysis with GLAD.

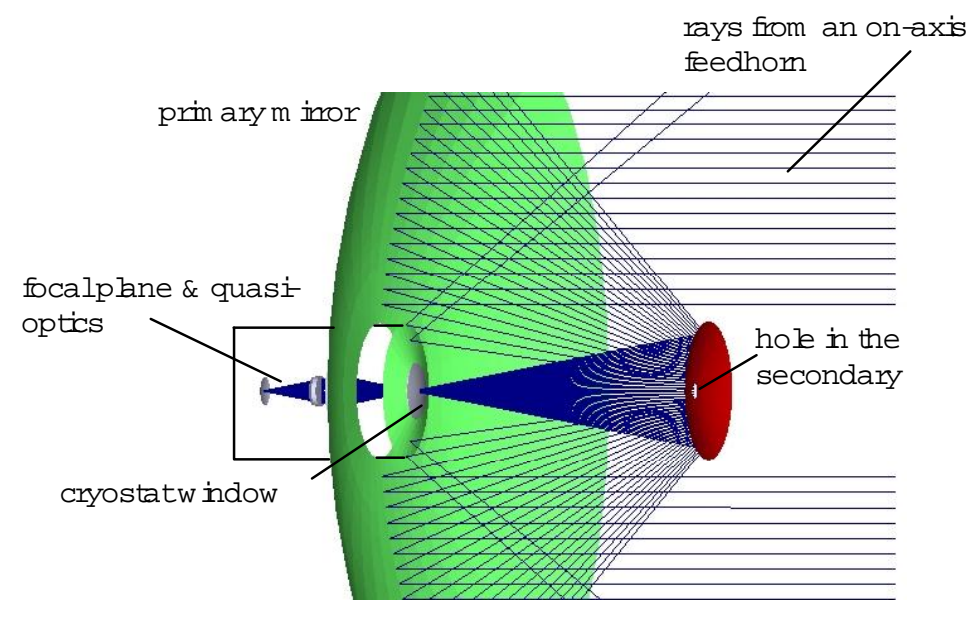

Fig. 1. Schematic diagram of the QUEST telescope optics.

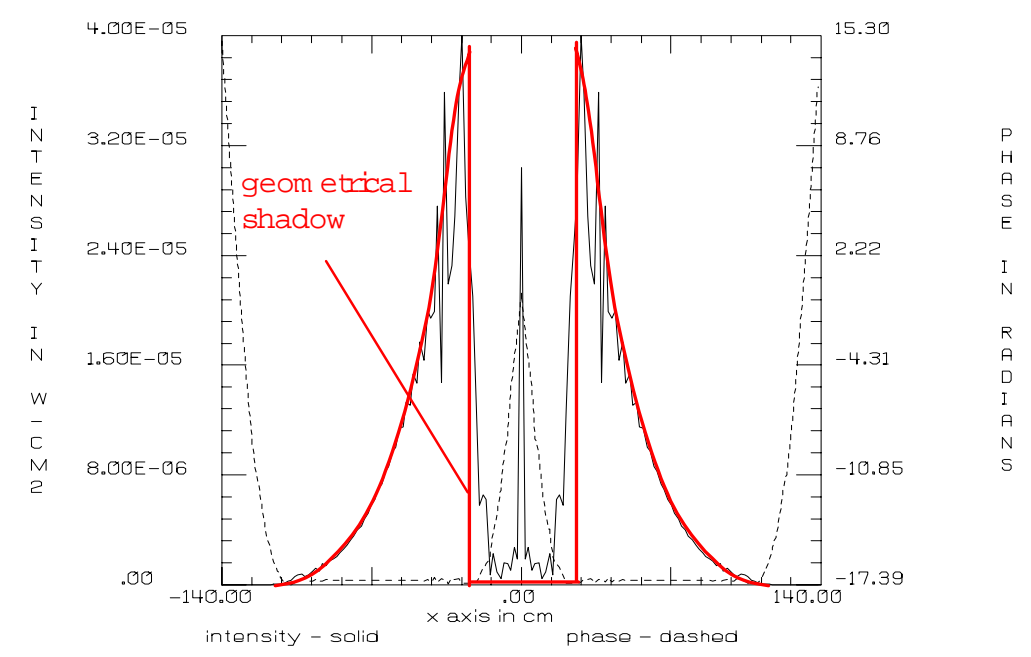

Fig. 2. GLAD prediction of the beam pattern at the primary mirror after being reflected from the secondary (see Fig. 1). $(\mathrm{r}=40 \mathrm{~mm}$ hole in the secondary, $\lambda=2.0 \mathrm{~mm})$. The horns were modelled as having a simple Gaussian profile. Although GLAD uses plane-wave decomposition, it treats fields as scalar fields and is therefore limited to paraxial systems.

However, care must also be taken with Fresnel diffraction and the paraxial mode approach. Fig. $3^{5}$ shows the near- and far-field intensity patterns for uniform plane-wave illumination of apertures of radius between $3 \lambda$ and $30 \lambda$. GLAD is taken as an example of a paraxial package and its results were closely matched by GBM, Zemax and CODE V. These examples were chosen so that an on-axis minimum is predicted using a simple Fresnel diffraction calculation. The paraxial packages do indeed produce the on-axis minimum indicating that they are correct within the limits of Fresnel diffraction. 
The software package GRASP, on the other hand, uses PO; that is, it calculates the field radiated by a reflector using an approximate surface current distribution determined from the incident magnetic field. It assumes that the field on that part of the reflector not directly illuminated by the incoming field is zero, appropriate where the radius of curvature of the reflector is many wavelengths. The Physical Theory of Diffraction (PTD) was developed to introduce currents associated with the edge into the assumed current distribution. Fig. 3 shows that there is a significant discrepancy between the beam patterns predicted using PO and Fresnel diffraction, particularly in the case of a small aperture. This is associated with the non-constant obliquity factor across the aperture. The approximations made by the paraxial packages are clearly not valid for short propagation distances. In the far field, by contrast, the angles associated with the obliquity factor are relatively small and one gets much closer agreement between the predictions of GRASP and the paraxial packages. There will be discrepancies if the beam spreads out into large (non-paraxial) angles. We found that these were not very significant for aperture diameters greater than $6 \lambda$. There is also the issue that, in far field calculations involving the paraxial approximation, it is assumed that $\theta \approx \sin \theta \approx \tan \theta$. In many situations a full PO analysis is prohibitively computationally intensive but Fresnel diffraction can be used as an alternative once these considerations are borne in mind.
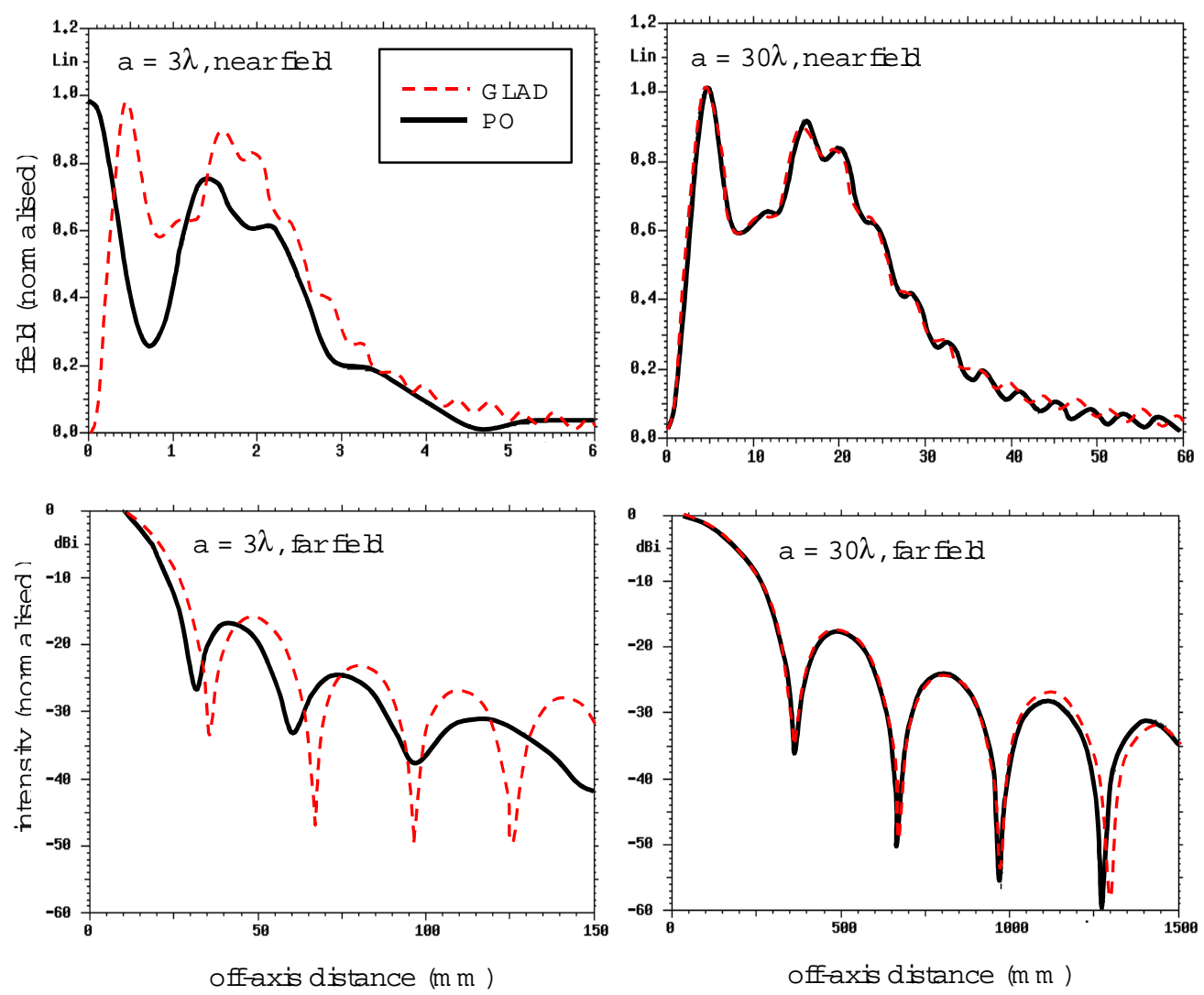

Fig. 3. Beam amplitude in the near $\left(\mathrm{z}_{\text {out }}=a^{2} / 4 \lambda\right)$ and far $\left(\mathrm{z}_{\text {out }}=20 a^{2} / \lambda\right)$ field of an aperture of radius $a=3 \lambda$ and $a=$ $30 \lambda$, calculated using GLAD and GRASP (physical optics). GLAD is taken to be representative of paraxial packages. $\lambda=1 \mathrm{~mm}$ in all cases. (see reference ${ }^{5}$ for details).

Typically we use variety of techniques to study any given $\mathrm{THz}$ system. In practice the usefulness of a software package or technique depends on factors such as the physical size of the system compared to the operating wavelength, the types of optical components used and whether the analysis is needed for the design or verification stage of a project. In particular, we have found that there is a lack of design tools in the $\mathrm{THz}$ regime and that we would like to have software that models a system using a range of methods and approximations. 
To this end we have begun work on a software package we call MODAL ${ }^{1}$ (Maynooth Optical Design and $\underline{A}$ nalysis Laboratory). MODAL can be used for the design and analysis of $\mathrm{THz}$ systems using PO (vector and scalar approximation) and Fresnel diffraction using Gaussian beam modes. Gaussian beam modes have long been used as a numerical technique for the analysis of long-wavelength systems but have been found to be very slow when modelling off-axis mirrors ${ }^{16}$, components which are common in compact quasi-optical systems such as HIFI and PLANCK. We have improved on the standard Gaussian beam mode analysis (GBMA) method by extending a powerful approach based on Singular Value Decomposition (SVD) ${ }^{17,18}$ to improve its efficiency when applied to complex optical surfaces. In the following sections we will describe the SVD technique in detail, describe the MODAL software package and present the results from our study of selected $\mathrm{THz}$ astronomical instruments.

\section{MODAL DECOMPOSITION USING SVD}

Gaussian beam modes constitute complete orthonomal sets that are each solutions to the paraxial wave equation. Any arbitrary solution of this equation can therefore be expressed as a superposition of Gaussian modes, the particular mode set, $\Psi_{i}$, being chosen as appropriate for the symmetry of the problem (e.g. Gauss-Hermite, Gauss-Laguerre);

$$
E(\boldsymbol{r})=E(x, y, z)=\sum_{i} A_{i} \Psi_{i}(x, y, z ; W(z), R(z)) e^{j\left(\Delta \phi_{i}(z)\right)}
$$

where $E(\boldsymbol{r})$ is the paraxial field, $A_{i}$ are the mode coefficients, $W(z)$ is the beam width parameter, $R(z)$ is the phase radius of curvature and $\Delta \phi_{i}$ is the phase slippage term. The choice of the optimum beam mode set is crucial to the efficiency of the Gaussian beam mode approach. Generally a source field can be represented to a high accuracy by the sum of only a few modes. Once the mode coefficients are known, it is straightforward to model the propagation of a beam by simply keeping track of the evolution of the beam width, the phase radius of curvature and the phase slippage between modes (using, for example, ABCD matrices).

If the field is known over the surface $S$ then the mode coefficients are determined by calculating the overlap integrals

$$
A_{i}=\int_{S} E(x, y, z) \Psi_{i}^{*}(x, y, z) d S .
$$

This decomposition of a field is the computationally intensive step but it only has to be carried out once if there is no scattering of power between modes (if mirrors and lenses are treated as perfect phase transformers with no truncation). However if an optical element introduces a significant amount of power scattering between modes, then the number of integrations needed to derive a scattering matrix in a straightforward way can be prohibitive. Indeed, if an off-axis mirror is treated as an inclined phase-transforming plane then it is necessary to determine the mode coefficients of the scattered field over a plane that is not orthogonal to the direction of propagation and therefore over which the mode set is not orthogonal ${ }^{16}$. These considerations have limited the efficiency with which GMBA has been applied to practical optical systems in all but preliminary analyses. A more economical numerical method for determining the expansion coefficients over complex surfaces is key to improving the efficiency of quasi-optical analyses using Gaussian beam modes.

An alternative to calculating overlap integrals is to attempt to fit a linear combination (coefficients $\tilde{\boldsymbol{A}}$ ) of the mode-set functions $\Psi i$ to the known field $\boldsymbol{E}$ at a limited number of sampled points $\boldsymbol{r}_{j}(j=1 \ldots M)$. Or, in a matrix formulation: 


$$
\widetilde{\boldsymbol{E}}=\left[\begin{array}{c}
\tilde{E}_{1} \\
\tilde{E}_{2} \\
\widetilde{E}_{3} \\
\vdots \\
\tilde{E}_{M}
\end{array}\right]=\left[\begin{array}{ccccc}
\Psi_{1,1} & \Psi_{1,2} & \Psi_{1,3} & \cdots & \Psi_{1, N} \\
\Psi_{2,1} & \Psi_{2,2} & \Psi_{2,3} & \cdots & \Psi_{2, N} \\
\Psi_{3,1} & \Psi_{3,2} & \Psi_{3,3} & \cdots & \Psi_{3, N} \\
\vdots & \vdots & \vdots & \ddots & \vdots \\
\Psi_{M, 1} & \Psi_{M, 2} & \Psi_{M, 3} & \cdots & \Psi_{M, N}
\end{array}\right]\left[\begin{array}{c}
\tilde{A}_{1} \\
\tilde{A}_{2} \\
\tilde{A}_{3} \\
\vdots \\
\tilde{A}_{N}
\end{array}\right]=\boldsymbol{\Psi} \tilde{\boldsymbol{A}},
$$

where $\tilde{E}_{j}=\tilde{E}\left(r_{j}\right)$ and $\Psi_{j, i}=\Psi_{i}\left(r_{j}\right)$.

If we minimize the residual of the solution $|\boldsymbol{E}-\tilde{\boldsymbol{E}}|$, the coefficients $\tilde{\boldsymbol{A}}_{i}$ approximate the accurate mode expansion coefficients $\tilde{\boldsymbol{A}}$. The usual choice for the norm of the residual

$$
|X|=\sum_{i=1}^{N}\left|X_{i}\right|^{2}
$$

reduces the determination of $\tilde{\boldsymbol{A}}$ to a linear least squares problem. The problem can be reformulated using the pseudoinverse (Moore-Penrose generalized inverse) $\Psi^{+}$of the mode matrix $\Psi$ as

$$
\tilde{\boldsymbol{A}}=\Psi^{+} \boldsymbol{E} .
$$

This solution can be shown to be the best approximation in the least squares sense as well as having the minimum vector norm. Singular value decomposition, or SVD, is one of the most powerful and efficient set of techniques for finding the pseudo inverse, and we have chosen to use it in our fit for the coefficients $\tilde{A}$ (see e.g. reference ${ }^{19}$ for details on implementing the method). SVD is based on the theorem that any $N \times M$ matrix $\boldsymbol{B}$ can be written as the product of an $N \times M$ column-orthogonal matrix $\boldsymbol{U}$, an $M \times M$ diagonal matrix $\boldsymbol{W}$ (whose elements are $\geq 0$ and termed the singular values) and the transpose of an $\mathrm{M} \times \mathrm{M}$ orthogonal matrix $\boldsymbol{V}$, that is

$$
\begin{aligned}
& \boldsymbol{B}^{+}=\boldsymbol{U} \boldsymbol{W} \boldsymbol{V}^{T}, \\
& W=\left(\begin{array}{ccccc}
w_{1} & 0 & 0 & \ldots & 0 \\
0 & w_{2} & 0 & \ldots & 0 \\
0 & 0 & w_{3} & \ldots & 0 \\
\vdots & \vdots & \vdots & \ddots & \vdots \\
0 & 0 & 0 & \ldots & w_{M}
\end{array}\right), \\
& \boldsymbol{U}^{T} \boldsymbol{U}=1 \\
& \boldsymbol{V}^{T} \boldsymbol{V}=1 .
\end{aligned}
$$

If $N<M$ then the singular values $w_{j}$ for $j=N+1, \ldots M$ and the corresponding columns of $\boldsymbol{U}$ are all zero (and eqns. 8 and 9 hold only for indices up to $N$ ).

If all the $w_{j}$ are non-zero, the pseudo-inverse $\boldsymbol{B}^{+}$of $\boldsymbol{B}$ is given by

$$
\boldsymbol{B}^{+}=\boldsymbol{V} \boldsymbol{W}^{-1} \boldsymbol{U}^{T}
$$

while if one or more of the $w_{j}$ are zero the best solution is given by

$$
\overline{\boldsymbol{B}}^{+}=\boldsymbol{V} \overline{\boldsymbol{W}}^{-1} \boldsymbol{U}^{T},
$$


where the inverse of the zero singular values are replaced with zeros:

$$
\overline{\boldsymbol{W}}^{-1}=\left(\begin{array}{ccccccc}
\frac{1}{w_{1}} & 0 & \ldots & 0 & 0 & \ldots & 0 \\
0 & \frac{1}{w_{2}} & \ldots & 0 & 0 & \ldots & 0 \\
\vdots & \vdots & \ddots & \vdots & \vdots & \ldots & \vdots \\
0 & 0 & \ldots & \frac{1}{w_{N}} & 0 & \ldots & 0 \\
0 & 0 & \ldots & 0 & 0 & \ldots & 0 \\
\vdots & \vdots & \vdots & \vdots & \vdots & \ddots & \vdots \\
0 & 0 & \ldots & 0 & 0 & \ldots & 0
\end{array}\right) .
$$

It often happens that some of the $w_{j}$ 's are very small but non-zero so that $\boldsymbol{W}$ is ill-conditioned. In this case their reciprocals should also be replaced by zero.

We use this SVD approach because it has many advantages over other techniques for solving least squares problems. It is generally numerically stable and can deal with both over- and under-determined problems. Of course, the selection of an appropriate set of modes ( $W$ and $R$ in eqn. 1) to represent the field accurately using as few modes as possible is not trivial, but the SVD technique does provide information that can be used to help in the selection. There are several approaches that can be taken to determine the optimum mode set and here we discuss two: choosing the mode set to maximising the power in the fundamental and choosing the mode set so that the effective spatial extent of the highest order mode corresponds to that of the field.

Take for example the field that results from plane-wave illumination of a circular aperture of radius $a$. Choosing $W=$ $0.891 a$ and $R=\infty$ maximises the lowest-order mode coefficient but the power in the higher-order modes, although small, is spread essentially over a large number of higher-order modes with the result that they are all required to accurately model the field. The choice of $W$ might more usefully take into account the ability of the highest-order mode to model the edge of the field. If we consider Gauss-Hermite modes, the final zero crossing of the $\mathrm{n}^{\text {th }}$ mode is approximately given by $\sqrt{0.75 N} W$. Matching this to the extent of the field to be decomposed gives an optimal $W=a / \sqrt{0.75 N}$. $R$ is again chosen to match the phase curvature of the field. Fig. 4 shows the reconstruction of a one-dimensional top-hat field using the two different choices of mode set. We use the latter in our SVD reconstructions.

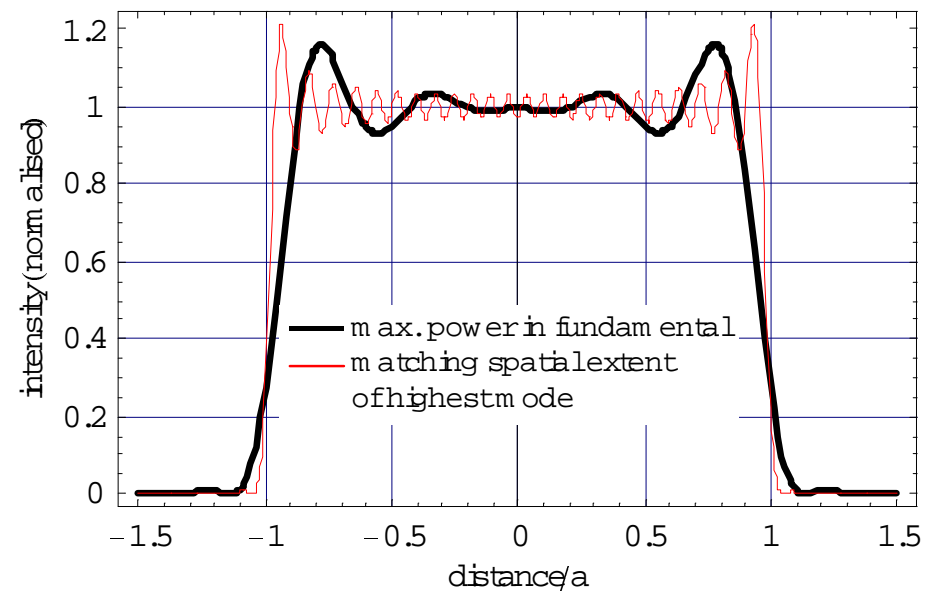

Fig. 4. Top-hat reconstruction using 50 Gauss-hermite modes with $W=1.01 a$ (maximizing the power in the fundamental for a $1 \mathrm{D}$ reconstruction) and $\mathrm{W}=a / \sqrt{0.75 \times 50 \mathrm{~W}} \cdot a$ is the radius of the field distribution. 
The number and locations of the field sampling points $r_{j}$ must also be given careful consideration. Nyquist's theorem says that sampling must be carried at intervals of $\lambda / 2$ or less. We have found that in order to determine the field using SVD fitting techniques it is important to use knowledge of the field beyond the edge of the scattering optical component. A straightforward way of achieving this in practice is to set the field equal to zero here (an approach consistent with the paraxial approximation). This padding out with zeros is only necessary up to a radius where all the power in the highest order modes is essentially negligible.

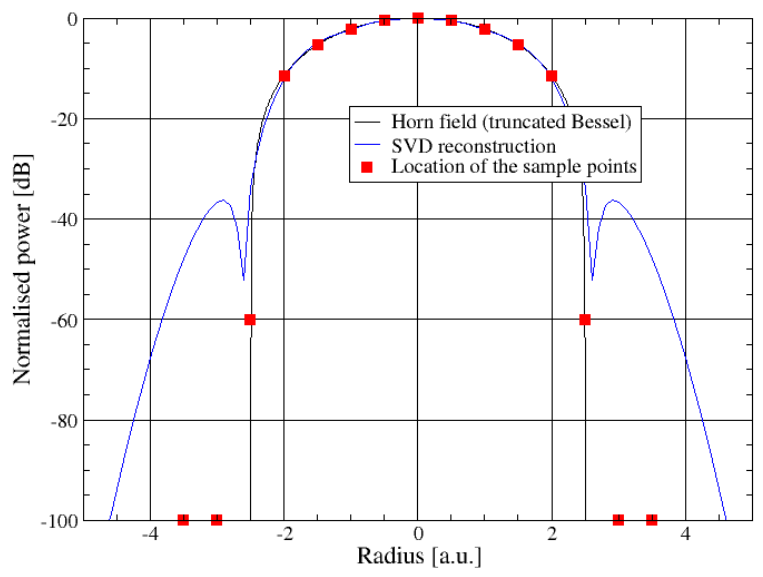

\begin{tabular}{|lc|l|l|}
\hline \multicolumn{2}{|c|}{$\begin{array}{c}\text { Mode } \\
m\end{array}$} & $\begin{array}{l}\text { O verlap } \\
\left|A_{m n}\right|^{2}\end{array}$ & $\mid \begin{array}{l}\text { SVD } \\
\left|A_{m n}\right|^{2}\end{array}$ \\
\hline 0 & 0 & 4.04409 & 4.04388 \\
\hline 0 & 2 & 0.55084 & 0.55008 \\
\hline 0 & 4 & 0.04844 & 0.04762 \\
\hline 0 & 6 & 0.00026 & 0.00054 \\
\hline 0 & 8 & 0.00302 & 0.00385 \\
\hline 2 & 2 & 0.03231 & 0.03163 \\
\hline 4 & 4 & 0.00155 & 0.00284 \\
\hline 6 & 6 & 0.00022 & 0.00059 \\
\hline 8 & 8 & 0.00004 & 0.00002 \\
\hline
\end{tabular}

Fig. 5. The SVD decomposition of a truncated Bessel function. A selection of the resulting mode coefficients are compared with those calculated using overlap integrals in the accompanying table. A total of $15 \times 15$ modes were used in the $2 \mathrm{D}$ reconstruction.

Fig. 5 shows the decomposition of a truncated Bessel function into Gauss-Hermite modes using SVD. The mode coefficients compare well with those determined using overlap integrals. (A truncated Bessel function is a good description of the field produced by a scalar horn.)

Once the best mode set and the corresponding coefficients have been determined for a source, the beam can be propagated through a quasi-optical system in the usual manner, reconstructing the field at each optical element which becomes the source for the next propagation step. The results of such an SVD analysis are shown in Fig. 7 for a test case consisting of a corrugated conical horn and two off-axis ellipsoidal mirrors (Fig. 6). The results are compared with those obtained using physical optics, both a scalar and full-vector calculation.

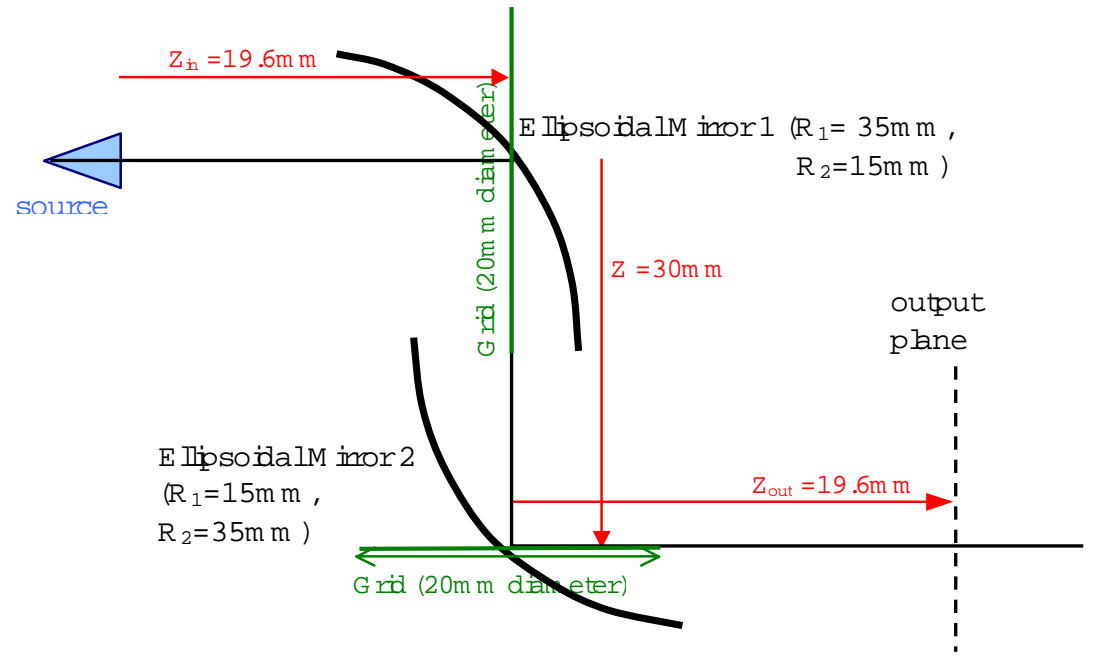

Fig. 6 Schematic drawing of the two-mirror test system. The source is a conical horn with an aperture radius of $2.5 \mathrm{~mm}$ and a slant length of $15.4 \mathrm{~mm}$. The source field is polarized perpendicular to the plane of the diagram. 

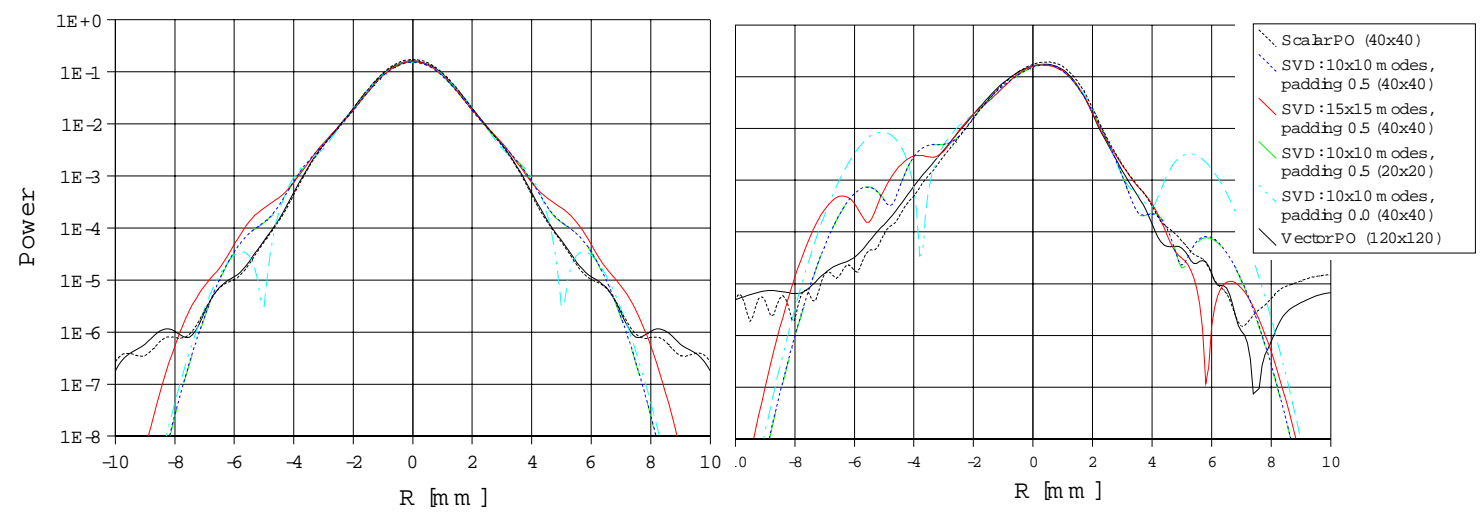

Fig. 7. Symmetric (left) and asymmetric (right) cuts across the output plane of the test system in Fig. 6. Power is normalized to the on-axis vector PO result. Different numbers of modes and grid points at the mirrors were used for SVD calculations. The source was defined on a $24 \times 24$ grid in each case and then further padded with zeros outside the field area. The number of extra zero points, expressed as a fraction of the main grid size, is given in the legend.

With the exception of the coarsest grid used $(10 \times 10$ example, not shown), we found that the physical optics results were well produced by the SVD technique down to below -20dB. The SVD calculations were significantly faster however, by a factor of up to 1000 depending on the particular parameters used. This is where the real advantage of the technique lies. The number of modes and the sampling used are important considerations and work is ongoing into how to make the best choice. The SVD technique described here is currently being incorporated into our design and analysis software, MODAL, discussed next.

\section{MAYNOOTH OPTICAL DESIGN AND ANALYSIS LABORATORY (MODAL)}

MODAL $^{1}$ combines an OpenGL-based user interface, for easy definition and manipulation of optical systems, with a powerful and flexible analysis engine that implements multiple propagation methods, ranging from plane wave decomposition to the full physical optics approach. The package provides export filters for analysis using external software. Calculations can be accelerated by running the code on a parallel computer composed of a heterogeneous collection of machines (using PVM). MODAL is by design a multi-platform package. It is currently being developed and tested in Windows and Linux.

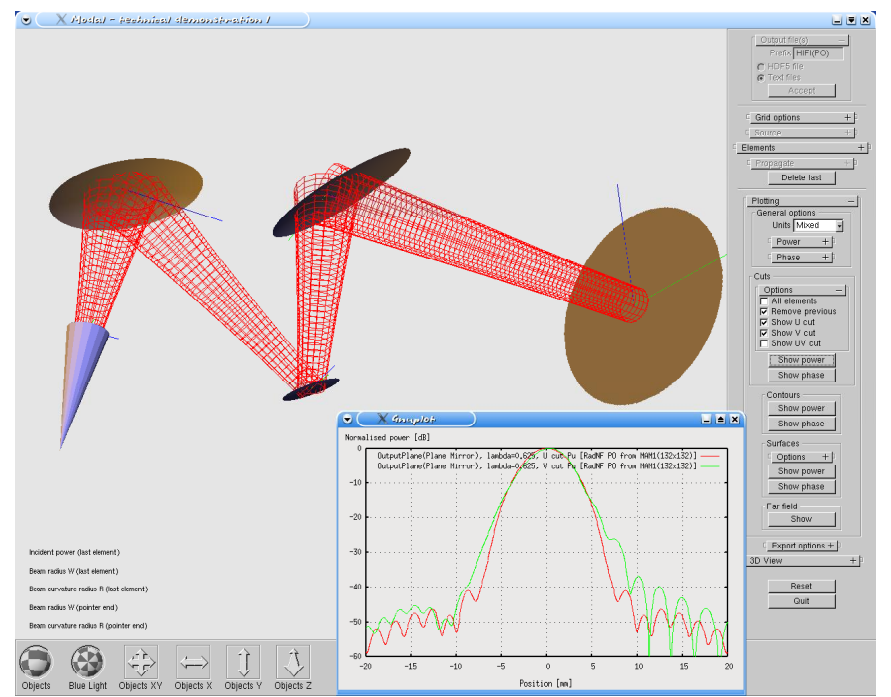

Fig. 8. MODAL graphical user interface showing a three-mirror test system (channel 1 Mixer Sub-Assembly of HIFI). 
The MODAL graphical user interface is shown in Fig. 8. MODAL keeps track of the best-fit Gaussian radius (plotted in Fig. 8), the phase radius of curvature and the waist positions to help in the design process. MODAL designs off-axis mirrors if the angle-of-throw and the input and output waist positions and sizes are specified. It is planned to further enhance the design capabilities of the package.

As an example, we have analysed the channel 1 mixer sub-assembly of HIFI $^{10}$ (Fig. 9) using MODAL's PO and SVD option. The predicted output intensity patterns are shown in Fig. 10.

Fig. 9. Geometry of the Channel 1 (480 - $640 \mathrm{GHz})$ Mixer SubAssembly (MSA) unit of the HIFI instrument ${ }^{10}$. It comprises a conical corrugated horn and three off-axis mirrors, two ellipsoidal (MAM2 \& MAM3) and one parabolic (MAM1). The system was simulated at $480 \mathrm{GHz}$ for one polarisation.
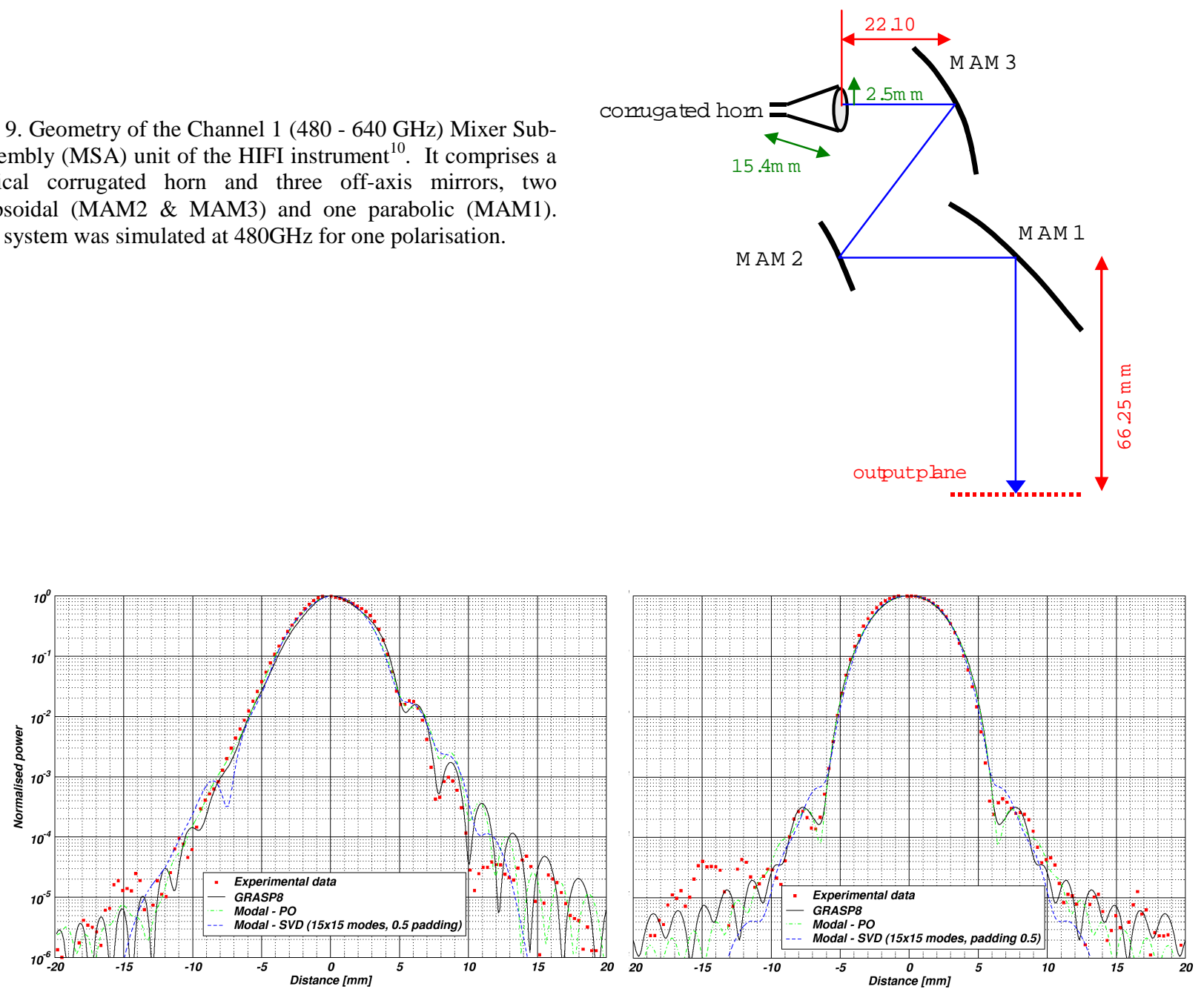

Fig. 10. Cuts of the HIFI MSA (Fig.9) output beam along the plane of symmetry (right) and asymmetry (left). The MODAL (PO and SVD) and GRASP8 predictions are shown.

Fig. 10 shows good agreement between the predictions made using the different analysis techniques. Experimental measurements ${ }^{10}$ are also shown. (There are still some differences in the mirror-rim definitions between GRASP/Experiment and MODAL.) The MODAL PO and SVD results match closely to -25dB or lower. 


\section{CONCLUSIONS \& FUTURE WORK}

We have described MODAL, a software package for quasi-optical design and analysis, currently under development at NUI Maynooth. The availability of a range of different analysis techniques in one package makes this an extremely flexible tool that will be of use throughout both the design and analysis phases of astronomical instrumentation at $\mathrm{THz}$ frequencies. We have briefly described a variety of different analysis techniques and shown examples using sections of the QUaD and Herschel HIFI instruments. A combination of techniques is typically used to model a complete system.

We believe that fast design and analysis methods based on SVD Gaussian Beam Mode decomposition are potentially very useful tools for quasi-optics. The SVD approach has proven useful in practical applications, even at this early stage of its development. In particular it can be used to quickly generate useful results during system design. Rigorous PO calculations can be prohibitively slow at this stage.

There are aspects of the method that need to be studied in greater detail before the SVD approach to modal analysis reaches sufficient maturity to be incorporated into MODAL as a reliable method of quasi-optical analysis. In the future we aim to address issues such as the optimization \& weighting of the field points sampled, the number and distribution of guard-band (zero) points and the best choice of mode set. The SVD method will also be extended to handle partially coherent fields $^{18}$. Code written for related work in NUI Maynooth, such as SCATTER ${ }^{20,21}$ (to predict the beam patterns of shaped corrugated horns) and FIRPOS $^{7}$ (fast physical optics code used for the analysis of PLANCK) can also be incorporated into MODAL.

\section{ACKNOWLEDGEMENTS}

We would like to thank NUI Maynooth, Enterprise Ireland and Science Foundation Ireland for supporting this work financially.

\section{REFERENCES}

1. http://www.may.ie/academic/physics/modal/modal.html

2. J.A.Murphy, A. Egan \& S. Withington: "Truncation efficiency in beam waveguides using Gaussian beam mode analysis," IEEE Trans. Antennas \& Propagation, 41, 1408-1413, 1993.

3. J.A.Murphy, S. Withington \& A. Egan: "Mode conversion at diffracting apertures in millimetre and submillimetre-wave optical systems," IEEE Trans. Microwave Theory and Techniques, 41, 1700-1702, 1993.

4. M.L. Gradziel, D. White, J.A. Murphy, S. Withington, "Improving the Efficiency of Quasi-optical Analysis and Design of Terahertz Systems", Proceedings of the 15th International Symposium on Space Terahertz Technology, Northampton, Massachusetts, USA, 2004.

5. O'Sullivan C., Atad-Ettedgui E., Duncan W., Henry D., Jellema W., Murphy J.A., Trappe N., van de Stadt H., Withington S. and Yassin G., "Far-IR Optics Design and Verification" Int. Journal IR \& Millimeter Waves, 23, 7, 2002.

6. G. Yassin, S. Withington, C. O'Sullivan, J. A. Murphy, T. Peacocke, W. Jellema and P. Wesselius "Electromagnetic modelling of Submillimetre-wave systems," Proceedings of the 13th International Symposium on Space Terahertz Technology, Harvard, USA, 2002.

7. V.B. Yurchenko, J.A. Murphy and J.-M. Lamarre, "Fast Physical Optics Simulations of the Multi-Beam DualReflector Submillimetre-Wave Telescope on the ESA PLANVK Surveyor", Int. J. Infrared and Millimeter Waves, 22, pp. 173-184, 2001.

8. S. Church, et al., "QUEST and DASI: A South-Pole CMB polarization experiment", New Astronomy Reviews, 47, 11-12, pp. 1083-1089, 2003.

9. T. de Graauw, F.P. Helmich, "Herschel-HIFI: The Heterodyne Instrument for the Far-Infrared", in The Promise of the Herschel Space Observatory, G. L. Pilbratt et al., eds., ESA SP-460, pp. 45-51, 2001.

10. W. Jellema, P. Wesselius, S. Withington, G. Yassin, J. A. Murphy, C. O’Sullivan, N. Trappe, T. Peacocke and B. Leone, "Experimental Verification of Electromagnetic Simulations of a HIFI Mixer Sub-Assembly", Proceedings of the $14^{\text {th }}$ International Symposium on Space Terahertz Technology, Tucson, Arizona, USA, 2003.

11. R.F. Harrington, Field computation by moments methods, Mac Millan, New York, 1968. 
12. G. Lawrence, "Optical system analysis with Physical Optics codes", SPIE no 766-18, O-E/Lase, 1987.

13.P.F. Goldsmith, Quasioptical Systems: Gaussian Beam Quasioptical Propagation and Applications, IEEE Press, 1998.

14. P.D. Einziger, S. Raz, and M. Shapira, J. Opt. Soc. Am. A, 3, 508-522 (1986).

15. M. Bowden et al., "Scientific optimization of a ground-based CMB polarization experiment", MNRAS, 349, 1, pp. 321-335, 2004.

16. J.A. Murphy, S. Withington, "Perturbation analysis of Gaussian-beam-mode scattering at off-axis ellipsoidal mirrors", Infrared Physics \& Technology, 37, 205-219, 1996.

17. A. Björk, Numerical Methods for Least Squares Problems, Society for Industrial and Applied Mathematics, Philadelphia, 1996.

18. S. Withington, M.P. Hobson, R.H. Berry, "Representing the behavior of partially coherent optical systems by using overcomplete basis sets", J. Opt. Soc. Am. A, 21, 207-217, 2004.

19. W.H. Press et al., Numerical Recipes in C, Cambridge University Press, 1992.

20. J. A. Murphy, R. Colgan, C. O'Sullivan, B. Maffei, \& P. Ade, "Radiation patterns of multi-moded corrugated horns for far-IR space applications", Infrared Physics \& Technology, 42, pp 515-528, 2001.

21. E. Gleeson, J.A. Murphy, S.E. Church, R. Colgan, C. O’Sullivan, "Electromagnetic Modelling of Few-Moded Winston Cones in the Far Infrared", in Proceedings of the Experimental Cosmology at millimeter wavelengths: 2K1BC Workshop, Breuil-Cervinia, Italy, July, 2001, M. De Petri and M. Gervasi. eds., AIP Conference Proceedings, Vol. 616, pp. 295-297, 2002. 